\title{
Stability and Time Delay Tolerance Analysis Approach for Networked Control Systems
}

\author{
Ashraf F. Khalil ${ }^{1}$ and Jihong Wang ${ }^{2}$ \\ ${ }^{1}$ Electrical and Electronic Engineering Department, University of Benghazi, Benghazi, Libya \\ ${ }^{2}$ School of Engineering, University of Warwick, Coventry CV4 7AL, UK \\ Correspondence should be addressed to Ashraf F. Khalil; ashraf.khalil@uob.edu.ly
}

Received 9 August 2014; Revised 27 October 2014; Accepted 29 October 2014

Academic Editor: Yun-Bo Zhao

Copyright (c) 2015 A. F. Khalil and J. Wang. This is an open access article distributed under the Creative Commons Attribution License, which permits unrestricted use, distribution, and reproduction in any medium, provided the original work is properly cited.

\begin{abstract}
Networked control system is a research area where the theory is behind practice. Closing the feedback loop through shared network induces time delay and some of the data could be lost. So the network induced time delay and data loss are inevitable in networked control Systems. The time delay may degrade the performance of control systems or even worse lead to system instability. Once the structure of a networked control system is confirmed, it is essential to identify the maximum time delay allowed for maintaining the system stability which, in turn, is also associated with the process of controller design. Some studies reported methods for estimating the maximum time delay allowed for maintaining system stability; however, most of the reported methods are normally overcomplicated for practical applications. A method based on the finite difference approximation is proposed in this paper for estimating the maximum time delay tolerance, which has a simple structure and is easy to apply.
\end{abstract}

\section{Introduction}

The key feature of networked control systems (NCSs) is that the information is exchanged through a network among control system components. So the network induced time delay is inevitable in NCSs. The time delay, either constant (up to jitter) or random, may degrade the performance of control systems and even destabilize the systems. NCSs can be defined as a control system where the control loop is closed through a real-time communication network [1]. The term networked control systems first appeared in Walsh's article in 1999 [2]. A typical organization of an NCS is shown in Figure 1. The reference input, plant output, and control input are exchanged through a real-time communication network. The main advantages of NCSs are modularity, simplified wiring, low cost, reduced weight, decentralization of control, integrated diagnosis, simple installation, quick and easy maintenance [3], and flexible expandability (easy to add/remove sensors, actuators, or controllers with low cost). NCSs are able to easily fuse global information to make intelligent decisions over large physical spaces which is important for many engineering systems such as the power system.
As the control loop is closed through a communication network the time delay and data dropout are unavoidable. Therefore networked control system can be regarded as a special case time delay system and many authors applied the time delay theorems to study NCSs [4]. Time delay, no doubt, increases complexity in analysis and design of NCSs. Conventional control theories built on a number of standing assumptions including synchronized control and nondelayed sensing and actuation must be reevaluated before they can be applied for NCSs [5].

The main goal of the most recent work is to reduce the conservativeness of the maximum time delay by using Lyapunov-Krasovskii functional with improved algorithms for solving the linear matrix inequalities (LMIs) set but with the expense of increasing complexity and computation time. Analytical and graphical methods have been studied in the literature (see, e.g., [6]). The stability criteria for NCSs based on Lyapunov-Krasovskii functional approach have been reported in [7-9]. In [7], a Lyapunov-Krasovskii function is used to derive a set of LMIs and the stability problem is generalized to a feasibility problem for the LMIs set. In many of the previously reported works, the controller is 


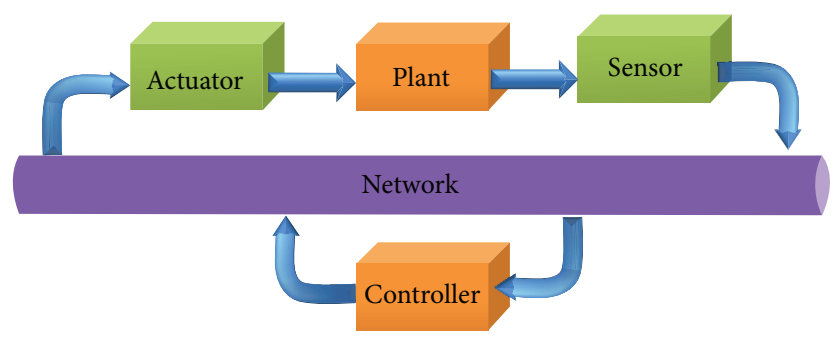

FIgURe 1: A typical networked control system.

designed in the absence of the time delay. In [10], an improved Lyapunov-Krasovskii function is used with triple integral terms. The LMI methods require the closed-loop system to be Hurwitz [8, 11, 12]. In [13], a modified cone complementary linearization algorithm based on the Lyapunov-Krasovskii approach is implemented. And the method reported in [14] is claimed to be less conservative and the computational complexity is reduced.

The authors in [15] derived an LMI-based method in the frequency domain, and then the LMI is transformed onto an equivalent nonfrequency domain LMI by applying Kalman-Yakubovich-Popov lemma. It has been reported in [16] that the ordinary Lyapunov stability analysis is linked by a suggested variable to state vectors through convolution and the stability analysis is simplified to only require solving a nonlinear algebraic matrix equation.

In [11], the hybrid system technique is used to derive a stability region. An upper bound is derived for time delay in an inequality form and the results are rather conservative. The hybrid system stability analysis technique has also been used in [17]. A simple analytical relation is derived between the sampling period, the time delay, and the controller gains. The same approach is used in [18] with more conservative stability region results. The model-based approach for deriving necessary and sufficient conditions for stability is presented in [19]. The stability criteria are derived in terms of the update time and the parameters of the model. The model-based approach is then extended to multiunits NCS in [20]. The optimal stochastic control was studied in [21] with a discrete-time system model where the random time delays are modeled using Markov chains and the controller uses the knowledge of the past state time delays by time stamping.

Most of the previously developed approaches require excessive load of computations, and also, for higher order systems, the load of computations will increase dramatically. In practice, engineers may find it difficult to apply those available methods in control system design because of the complexity of the methods and lack of guideline in linking between the design parameters and the system performance. Almost all the design procedures highly depend on the postdesign simulation to determine the design parameters. So there is a demand for a simple design approach with clear guidance for practical applications. In this paper, a new stability analysis and control design method is proposed, in which the design approach is simple and a clear design procedure is given.
The paper starts from the mathematical model of NCS and then the proposed method for estimating the maximum allowable delay bound is briefly described. A few examples are illustrated and the results are compared with those previously published in the literature. The cart and inverted pendulum problem is used to study the effect of the parameters on the maximum allowable delay bound.

\section{Mathematical Analysis}

Although the issues involved with time delays in control systems have been studied for a long time, it is difficult to find a method simple enough to be accepted by control system design engineers. It is found that the most previously reported methods rely on LMI techniques and they are generally too complicated for practical engineers to use and also involve heavy load of numerical calculations and computing time. The paper proposes a new method which has a simple structure and is used for estimating the maximum time delay allowed while the system stability can still be maintained. In most control systems the sampling time is preferred to be small [22]. The maximum allowable delay bound (MADB) can be defined as the maximum sampling period that guarantees the stability even with poor system performance. A continuous time-invariant linear system is shown in Figure 2 and given by

$$
\begin{aligned}
& \dot{\mathbf{x}}(t)=\mathbf{A} \mathbf{x}(t)+\mathbf{B u}(t), \\
& \mathbf{y}(t)=\mathbf{C} \mathbf{x}(t)+\mathbf{D} \mathbf{u}(t),
\end{aligned}
$$

where $\mathbf{x}(t) \in \mathfrak{R}^{n}$ is the system state vector, $\mathbf{u}(t) \in \mathfrak{R}^{m}$ is the system control input, $\mathbf{y}(t) \in \mathfrak{R}^{p}$ is the system output, and $\mathbf{A} \in \mathfrak{R}^{n \times n}, \mathbf{B} \in \mathfrak{R}^{n \times m}, \mathbf{C} \in \mathfrak{R}^{p \times n}$, and $\mathbf{D} \in \mathfrak{R}^{p \times m}$ are constant matrices with appropriate sizes.

Suppose that the control signals are connected to the control plant through a kind of network, so the time delay is inevitable to be involved in the feedback loop. The state feedback is therefore can be written as

$$
\mathbf{u}(t)=\mathbf{K} \mathbf{x}\left(t-\tau_{s c}-\tau_{c}-\tau_{c a}\right),
$$

where $\tau_{s c}$ is the time delay between the sensor and the controller, $\tau_{c}$ is the time delay in the controller, and $\tau_{c a}$ is the time delay from the controller to the actuator. $\mathbf{K}$ represents the feedback control gains with appropriate size. From (2) the networked control system can be modeled where the time delay is lumped between the sensor and the controller as shown in Figure 3.

The time delay may be constant, variable, or even random. In NCSs, the time delay is composed of the time delay from sensors to controllers, time delay in the controller, and controllers to actuators time delay which is given by

$$
\tau=\tau_{s c}+\tau_{c}+\tau_{c a} .
$$

For a general formulation the packet dropouts can be incorporated in (3) as follows:

$$
\tau=\tau_{s c}+\tau_{c}+\tau_{c a}+d h,
$$




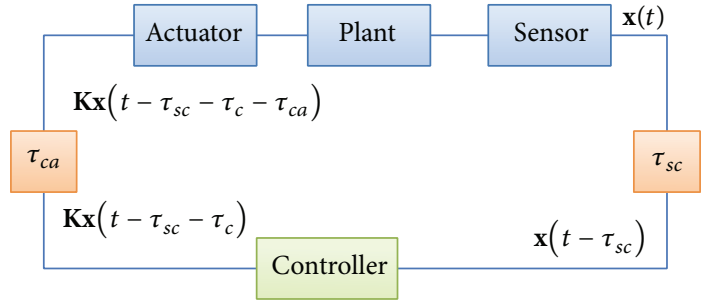

FIGURE 2: A networked control system with the time delay both from the sensor to the controller and from the controller to the actuator.

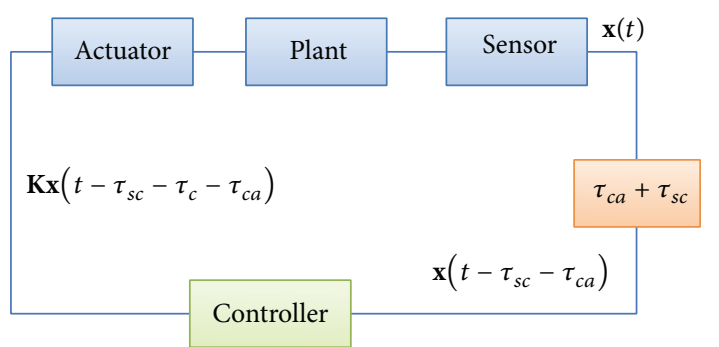

FIgURE 3: A simplified model of the networked control system.

where $d$ is the number of dropouts and $h$ the sampling period. And by (4) the data dropouts can be considered as a special case of time delay $[23,24]$. It is supposed that the following hypotheses hold.

Hypothesis 1 (H.1). (i) The sensors are clock driven. (ii) The controllers and the actuators are event driven. (iii) The data are transmitted as a single packet. (iv) The old packets are discarded. (v) All the states are available for measurements and hence for transmission.

Hypothesis 2 (H.2). The time delay $\tau$ is small to be less than one unit of its measurement.

Definition 1(D.1). For a function $f(t)$, the $n$th order reminder for its Taylor's series expansion is defined by

$$
R_{n}(f(t), \tau)=\sum_{n}^{\infty} \frac{f^{(n)}(\xi)}{n !} \tau^{n} .
$$

Applying the state feedback proposed in (2) to the system (1), we have

$$
\dot{\mathbf{x}}(t)=\mathbf{A x}(t)+\mathbf{B K} \mathbf{x}(t-\tau) .
$$

From (6), the following can be derived:

$$
\dot{\mathbf{x}}(t)=(\mathbf{A}+\mathbf{B K}) \mathbf{x}(t)+\mathbf{B K}[\mathbf{x}(t-\tau)-\mathbf{x}(t)] .
$$

Theorem 2. Suppose that (H.1) and (H.2) hold. For system (1) with the feedback control of (2), the closed-loop system is globally asymptotically stable if $\lambda_{i}(\Psi) \in C^{-}$, for $i=1,2, \ldots, n$ and all the state variables' 2 nd order reminders are small enough for the given value of $\tau$, where $\Psi$ is given by

$$
\Psi=\left[(I+\tau \mathbf{B K})^{-1}(\mathbf{A}+\mathbf{B K})\right] .
$$

Proof. The expression for $\mathbf{x}(t-\tau)$ can be obtained by Taylor expansion as

$$
\mathbf{x}(t-\tau)=\sum_{n=0}^{\infty}(-1)^{n} \frac{\tau^{n}}{n !} \mathbf{x}^{(n)}(t),
$$

where $\mathbf{x}^{(n)}(t)$ is the $n$th order derivative. The first order approximation of the delay term is given by

$$
\begin{aligned}
& \mathbf{x}(t-\tau)=\mathbf{x}(t)-\tau \dot{\mathbf{x}}(t)+\left(\frac{\tau^{2}}{2}\right) \ddot{\mathbf{x}}(t)+\mathbf{R}_{3}(\mathbf{x}, \tau), \\
& \mathbf{x}(t-\tau) \approx \mathbf{x}(t)-\tau \dot{\mathbf{x}}(t)+\left(\frac{\tau^{2}}{2}\right) \ddot{\mathbf{x}}(t), \\
& \mathbf{x}(t-\tau)=\mathbf{x}(t)-\tau \dot{\mathbf{x}}(t)+\mathbf{R}_{2}(\mathbf{x}, \tau) .
\end{aligned}
$$

From (10) it can be seen that $\mathbf{R}_{2}(x, \tau)$ depends on the time delay, $\tau$, and the higher order derivatives of $\mathbf{x}(t)$ which can be neglected if the time delay and the norm of $\mathbf{R}_{2}(x, \tau)$ are small. Then we have

$$
\mathbf{x}(t-\tau) \approx \mathbf{x}(t)-\tau \dot{\mathbf{x}}(t) .
$$

The assumption in (11) can be used without significant error, and this can be true for the following reasons. Firstly, the time delay in a computer network is very small in order of milli- or microseconds and at the worst few tenths of the second. Secondly, in most of the real control system applications the linearized model is used and the higher order terms are already neglected. Additionally, the higher order derivatives will be multiplied by $\tau^{n} / n$ which is much more smaller than $\tau$ because $\tau \ll 1$. Substituting (11) into (7), the following can be derived:

$$
\begin{aligned}
\dot{\mathbf{x}}(t) & \approx(\mathbf{A}+\mathbf{B K}) \mathbf{x}(t)-\tau \mathbf{B K} \dot{\mathbf{x}}(t), \\
\dot{\mathbf{x}}(t) & \approx\left[(\mathbf{I}+\tau \mathbf{B K})^{-1}(\mathbf{A}+\mathbf{B K})\right] \mathbf{x}(t), \\
\Psi & =\left[(\mathbf{I}+\tau \mathbf{B K})^{-1}(\mathbf{A}+\mathbf{B K})\right] .
\end{aligned}
$$

The system (13) will be globally asymptotically stable if

$$
\lambda_{i}(\Psi) \in \mathrm{C}^{-}, \quad \text { for } i=1,2, \ldots, n .
$$

Corollary 3. Suppose (H.1) and (H.2) hold. For the control system (1) with the control law (2), the closed-loop system is globally asymptotically stable if

$$
\tau<\frac{1}{\|\mathbf{B K}\|} .
$$

Proof. For system (1), suppose that the state feedback has been designed to ensure $\lambda(\mathbf{A}+\mathbf{B K}) \in \mathbf{C}^{-}$. Therefore, for a chosen positive definite matrix $\mathbf{P}=\mathbf{P}^{\mathrm{T}}$, it will find a positive definite matrix $\mathbf{Q}=\mathbf{Q}^{\mathrm{T}}$ to have

$$
\mathbf{P}(\mathbf{A}+\mathbf{B K})+(\mathbf{A}+\mathbf{B K})^{\mathrm{T}} \mathbf{P}=-\mathbf{Q} .
$$


Choose a Lyapunov functional candidate as

$$
\mathbf{V}(x)=\mathbf{x}^{\mathrm{T}} \mathbf{P} \mathbf{x}>0 \quad \forall \mathbf{x} \neq \mathbf{0} .
$$

The objective for the next step is to find the range of $\tau$ that will ensure $(\dot{\mathrm{V}}(x)<0 \forall \mathbf{x} \neq \mathbf{0})$ [25-27]. Taking the derivative of (18),

$$
\begin{gathered}
\dot{\mathbf{V}}(x)=\dot{\mathbf{x}}^{\mathrm{T}} \mathbf{P} \mathbf{x}+\mathbf{x}^{\mathrm{T}} \mathbf{P} \dot{\mathbf{x}} \\
\approx \mathbf{x}^{\mathrm{T}}\left[(\mathbf{A}+\mathbf{B K})^{\mathrm{T}} \mathbf{P} \mathbf{P}^{-\mathbf{1}}(\mathbf{I}+\tau \mathbf{B K})^{-\mathrm{T}} \mathbf{P}\right. \\
\left.\quad+\mathbf{P}(\mathbf{I}+\tau \mathbf{B K})^{-1} \mathbf{P}^{-\mathbf{1}} \mathbf{P}(\mathbf{A}+\mathbf{B K})\right] \mathbf{x} \\
-\mathbf{x}^{\mathrm{T}}\left[\mathbf{P}(\mathbf{A}+\mathbf{B K})+(\mathbf{A}+\mathbf{B K})^{\mathrm{T}} \mathbf{P}\right] \mathbf{x} \\
+\mathbf{x}^{\mathrm{T}}\left[\mathbf{P}(\mathbf{A}+\mathbf{B K})+(\mathbf{A}+\mathbf{B K})^{\mathrm{T}} \mathbf{P}\right] \mathbf{x} \\
\approx \mathbf{x}^{\mathrm{T}}\left[(\mathbf{A}+\mathbf{B K})^{\mathrm{T}} \mathbf{P} \mathbf{P}^{-\mathbf{1}}(\mathbf{I}+\tau \mathbf{B K})^{-\mathrm{T}} \mathbf{P}\right. \\
\quad-(\mathbf{A}+\mathbf{B K})^{\mathrm{T}} \mathbf{P}+\mathbf{P}(\mathbf{I}+\tau \mathbf{B K})^{-1} \mathbf{P}^{-\mathbf{1}} \mathbf{P}(\mathbf{A}+\mathbf{B K}) \\
\quad-\mathbf{P}(\mathbf{A}+\mathbf{B K})] \mathbf{x}-\mathbf{x}^{\mathrm{T}} \mathbf{Q} \mathbf{x} .
\end{gathered}
$$

Rearranging the terms in the above equation, then

$$
\begin{aligned}
\dot{\mathbf{V}}(\mathbf{x}) \approx & \mathbf{x}^{\mathrm{T}}\left\{(\mathbf{A}+\mathbf{B K})^{\mathrm{T}} \mathbf{P}\left[\mathbf{P}^{-\mathbf{1}}(\mathbf{I}+\tau \mathbf{B K})^{-\mathrm{T}} \mathbf{P}-\mathbf{I}\right]\right. \\
& \left.+\left[\mathbf{P}(\mathbf{I}+\tau \mathbf{B K})^{-1} \mathbf{P}^{-1}-\mathbf{I}\right] \mathbf{P}(\mathbf{A}+\mathbf{B K})\right\} \mathbf{x} \\
& -\mathbf{x}^{\mathrm{T}} \mathbf{Q} \mathbf{x} .
\end{aligned}
$$

If $\mathbf{P}(\mathbf{I}+\tau \mathbf{B K})^{-\mathbf{1}} \mathbf{P}^{-\mathbf{1}}-\mathbf{I}=\mathbf{I}$ then (20) will become

$$
\mathbf{x}^{\mathrm{T}}\left[\mathbf{P}(\mathbf{A}+\mathbf{B K})+(\mathbf{A}+\mathbf{B K})^{\mathrm{T}} \mathbf{P}\right] \mathbf{x}-\mathbf{x}^{\mathrm{T}} \mathbf{Q} \mathbf{x}=0 .
$$

Move the last term to the right hand side; the following will be derived:

$$
\mathbf{x}^{\mathrm{T}}\left[\mathbf{P}(\mathbf{A}+\mathbf{B K})+(\mathbf{A}+\mathbf{B K})^{\mathrm{T}} \mathbf{P}\right] \mathbf{x}=\mathbf{x}^{\mathrm{T}} \mathbf{Q} \mathbf{x} .
$$

So $\left\|\mathbf{P}(\mathbf{A}+\mathbf{B K})+(\mathbf{A}+\mathbf{B K})^{\mathbf{T}} \mathbf{P}\right\| \cdot\|\mathbf{x}\|^{2}=\|\mathbf{Q}\| \cdot\|\mathbf{x}\|^{2}$.

Assuming that we can find a positive number to make the following hold:

$$
\left\|\mathbf{P}(\mathbf{A}+\mathbf{B K})+(\mathbf{A}+\mathbf{B K})^{\mathbf{T}} \mathbf{P}\right\|=2 \gamma\left\|(\mathbf{A}+\mathbf{B K})^{\mathbf{T}} \mathbf{P}\right\|=\|\mathbf{Q}\|
$$

then $\gamma$ can be considered as the norm of $\mathbf{P}^{-\mathbf{1}}(\mathbf{I}+\tau \mathbf{B K})^{-\mathbf{1}} \mathbf{P}-\mathbf{I}$. Therefore, we have

$$
\begin{aligned}
\mathbf{x}^{\mathbf{T}}\left[(\mathbf{A}+\mathbf{B K})^{\mathrm{T}} \mathbf{P}\left[\mathbf{P}^{-\mathbf{1}}(\mathbf{I}+\tau \mathbf{B K})^{-\mathbf{T}} \mathbf{P}-\mathbf{I}\right]\right. \\
\left.\quad+\left[\mathbf{P}(\mathbf{I}+\tau \mathbf{B K})^{-1} \mathbf{P}^{-\mathbf{1}}-\mathbf{I}\right] \mathbf{P}(\mathbf{A}+\mathbf{B K})\right] \mathbf{x} \\
\leq 2\left\|\left(\mathbf{P}^{-\mathbf{1}}(\mathbf{I}+\tau \mathbf{B K})^{-\mathbf{T}} \mathbf{P}-\mathbf{I}\right) \mathbf{P}(\mathbf{A}+\mathbf{B K})\right\| \cdot\|\mathbf{x}\|^{2} .
\end{aligned}
$$

Choose

$$
\left\|\mathbf{P}^{-\mathbf{1}}(\mathbf{I}+\tau \mathbf{B K})^{-1} \mathbf{P}-\mathbf{I}\right\| \leq 1 .
$$

Use Neumann series formula for the inverse of the sum of two matrices:

$$
\begin{aligned}
& (\mathbf{I}+\tau \mathbf{B K})^{-1} \\
& \quad=\mathbf{I}-\tau \mathbf{B K}+\tau^{2}(\mathbf{B K})^{2}-\tau^{3}(\mathbf{B K})^{3}+\cdots-.
\end{aligned}
$$

For small time delays $\tau \ll 1(26)$ can be given as

$$
(\mathbf{I}+\tau \mathbf{B K})^{-1} \approx \mathbf{I}-\tau \mathbf{B K} .
$$

Applying (27) into (25) then we have

$$
\begin{aligned}
& \left\|\mathbf{P}^{-\mathbf{1}}(\mathbf{I}+\tau \mathbf{B K})^{-1} \mathbf{P}-\mathbf{I}\right\| \\
& \quad \approx\left\|\mathbf{P}^{-\mathbf{1}}(\mathbf{I}-\tau \mathbf{B K}) \mathbf{P}-\mathbf{I}\right\|=\|\tau \mathbf{B K}\|<1 .
\end{aligned}
$$

And finally we get

$$
\tau<\frac{1}{\|\mathbf{B K}\|} .
$$

That is, for any $\tau<1 /\|\mathbf{B K}\|, \dot{\mathrm{V}}(x)<0$, the system will be globally asymptotically stable.

Theorem 2 and Corollary 3 give us a simple tool in estimating the maximum allowable time delay for NCSs. Further analysis in the frequency domain is described below. Taking Laplace transform of (12), we have

$$
\begin{gathered}
s \mathbf{X}(s)=(\mathbf{A}+\mathbf{B K}) \mathbf{X}(s)-\tau s \mathbf{B K X}(s), \\
{[s \mathbf{I}-(\mathbf{A}+\mathbf{B K})+\tau s \mathbf{B K}] \mathbf{X}(s)=0 .}
\end{gathered}
$$

The characteristics equation is defined as

$$
[s \mathbf{I}-(\mathbf{A}+\mathbf{B K})+\tau s \mathbf{B K}]=0 .
$$

For a stable system the roots of the characteristics equation (31) must lie in the left hand side of the $s$-plane. From the characteristics equation, it is clear that the term $\tau s \mathbf{B K}$ influences the system performance and the stability as the term of $\tau$ s BK may push the closed-loop system poles toward the right hand side of the $s$-plane.

As we have seen the system characteristic is determined by the term $\tau \mathbf{B K} \dot{\mathbf{x}}(t)$ in a certain level. This term can be regarded as a differentiator in the feedback loop, so it will introduce extra zeros to the closed-loop system and the time delay can be considered to have resulted in a variable gain to the feedback path. For more accurate estimation the second or third-order difference approximation can be used as follows:

$$
\begin{gathered}
{\left[s \mathbf{I}-(\mathbf{A}+\mathbf{B K})+\tau s \mathbf{B K}-\frac{\tau^{2} s^{2}}{2} \mathbf{B K}\right]=0,} \\
{\left[s \mathbf{I}-(\mathbf{A}+\mathbf{B K})+\tau s \mathbf{B K}-\frac{\tau^{2} s^{2}}{2} \mathbf{B K}+\frac{\tau^{3} s^{3}}{6} \mathbf{B K}\right]=0 .}
\end{gathered}
$$

In the following a simple corollary for estimating the MADB in single-input-single-output NCS will be derived. 
Corollary 4. Suppose that (H.1) and (H.2) hold. The system (2) with the controller (3) is asymptotically stable if

$$
\tau<\frac{1}{\left|\lambda_{\min }(\mathbf{B K})\right|} .
$$

Proof. The main assumption is that the eigenvalues of the compensator, $\mathbf{B K}$, are all negative, $s_{1}<0, \ldots, s_{n}<0$, and are given by

$$
\mathbf{B K}-s \mathbf{I}_{n \times n}=\left[\begin{array}{cccc}
a_{11}-s & a_{12} & \cdots & a_{1 n} \\
a_{21} & a_{22}-s & \cdots & a_{2 n} \\
\vdots & \vdots & \ddots & \vdots \\
a_{n 1} & a_{n 2} & \cdots & a_{n n}-s
\end{array}\right]
$$

The characteristic equation is the determinant of (34). Assume that the eigenvalues are given by

$$
\begin{gathered}
s_{1}=\alpha_{1}, \ldots, s_{n}=\alpha_{n}, \\
\alpha_{1}<0, \ldots, \alpha_{n}<0 .
\end{gathered}
$$

Preliminary 1 (inverse eigenvalues theorem [28]). Given a matrix $\mathbf{X}$ that is nonsingular, with eigenvalues $\lambda_{1}, \ldots, \lambda_{n}>$ $0, \lambda_{1}, \ldots, \lambda_{n}$ are eigenvalues of $\mathbf{X}$ if and only if $\lambda_{1}{ }^{-1}, \ldots, \lambda_{n}{ }^{-1}$ are eigenvalues of $\mathbf{X}^{-1}$.

The eigenvalues of $\left(\mathbf{I}_{n \times n}+\tau \mathbf{B K}\right)$ are given by

$$
\begin{aligned}
\tau & \cdot \mathbf{B K}+\mathbf{I}_{n \times n}-\lambda \mathbf{I}_{n \times n} \\
= & {\left[\begin{array}{cccc}
\tau a_{11}+1-\lambda & \tau a_{12} & \cdots & \tau a_{1 n} \\
\tau a_{21} & \tau a_{22}+1-\lambda & \cdots & \tau a_{2 n} \\
\vdots & \vdots & \ddots & \vdots \\
\tau a_{n 1} & \tau a_{n 2} & \cdots & \tau a_{n n}+1-\lambda
\end{array}\right], }
\end{aligned}
$$

$\Delta\left(\tau \cdot \mathbf{B K}+\mathbf{I}_{n \times n}-\lambda \mathbf{I}_{n \times n}\right)$

$=\operatorname{det}\left(\left[\begin{array}{cccc}\tau a_{11}+1-\lambda & \tau a_{12} & \cdots & \tau a_{1 n} \\ \tau a_{21} & \tau a_{22}+1-\lambda & \cdots & \tau a_{2 n} \\ \vdots & \vdots & \ddots & \vdots \\ \tau a_{n 1} & \tau a_{n 2} & \cdots & \tau a_{n n}+1-\lambda\end{array}\right]\right)$,

$\Delta\left(\tau \cdot \mathbf{B K}+\mathbf{I}_{n \times n}-\lambda \mathbf{I}_{n \times n}\right)$

$$
=\tau^{n} \operatorname{det}\left(\left[\begin{array}{cccc}
a_{11}+\frac{1-\lambda}{\tau} & a_{12} & \cdots & a_{1 n} \\
a_{21} & a_{22}+\frac{1-\lambda}{\tau} & \cdots & a_{2 n} \\
\vdots & \vdots & \ddots & \vdots \\
a_{n 1} & a_{n 2} & \cdots & a_{n n}+\frac{1-\lambda}{\tau}
\end{array}\right]\right)
$$

Replacing $(1-\lambda) / \tau$ by $-s$ in (37) we get

$$
=\tau^{n} \operatorname{det}\left(\left[\begin{array}{cccc}
a_{11}-s & a_{12} & \cdots & a_{1 n} \\
a_{21} & a_{22}-s & \cdots & a_{2 n} \\
\vdots & \vdots & \ddots & \vdots \\
a_{n 1} & a_{n 2} & \cdots & a_{n n}-s
\end{array}\right]\right)=0 .
$$

Solving (38) the eigenvalues are given as

$$
\begin{gathered}
\frac{\left(\lambda_{1}-1\right)}{\tau}=\alpha_{1}, \ldots, \frac{\left(\lambda_{n}-1\right)}{\tau}=\alpha_{n}, \\
\alpha_{1}<0, \ldots, \alpha_{n}<0, \\
\lambda_{1}=1+\tau \alpha_{1}, \ldots, \lambda_{n}=1+\tau \alpha_{n}, \\
\alpha_{1}<0, \ldots, \alpha_{n}<0 .
\end{gathered}
$$

If $\tau<1 /\left|\alpha_{\max }\right|$ then all the eigenvalues are positive and the system is asymptotically stable, and if $\tau>1 /\left|\alpha_{\max }\right|$ at least one of the eigenvalues will be negative then.

If $\tau<1 /\left|\lambda_{\min }(\mathbf{B K})\right|$ and (H.1) and (H.2) hold then the system is asymptotically stable.

Corollary 5. Suppose that (H.1) and (H.2) hold. For system (1) with the control law (2), the closed-loop system is globally asymptotically stable if

$$
\tau<\frac{1}{a b s(\mathbf{K B})} \quad \text { (where abs is the absolute value). }
$$

From Preliminary 1, the signs of the eigenvalues of $\left(\mathbf{I}_{n \times n}+\right.$ $\tau \mathbf{B K})^{-1}$ and $\left(\mathbf{I}_{n \times n}+\tau \mathbf{B K}\right)$ are the same. For a single-inputsingle-output control system the matrix $\mathbf{B K}$ can be written as

$$
\mathbf{B K}=\left[\begin{array}{c}
b_{1} \\
b_{2} \\
\vdots \\
b_{n}
\end{array}\right]\left[\begin{array}{llll}
k_{1} & k_{2} & \cdots & k_{n}
\end{array}\right]=\left[\begin{array}{cccc}
b_{1} k_{1} & b_{1} k_{2} & \cdots & b_{1} k_{n} \\
b_{2} k_{1} & b_{2} k_{2} & \cdots & b_{2} k_{n} \\
\vdots & \vdots & \ddots & \vdots \\
b_{n} k_{1} & b_{n} k_{2} & \cdots & b_{n} k_{n}
\end{array}\right] .
$$

The interesting property of $\mathbf{B K}$ is that it is singular. The eigenvalues of $\mathbf{B K}$ are given by

$$
\mathbf{B K}-\lambda \mathbf{I}_{n \times n}=\left[\begin{array}{cccc}
b_{1} k_{1}-\lambda & b_{1} k_{2} & \cdots & b_{1} k_{n} \\
b_{2} k_{1} & b_{2} k_{2}-\lambda & \cdots & b_{2} k_{n} \\
\vdots & \vdots & \ddots & \vdots \\
b_{n} k_{1} & b_{n} k_{2} & \cdots & b_{n} k_{n}-\lambda
\end{array}\right] .
$$

The characteristics equation of $\mathbf{B K}$ is the determinant of (42) and is given by

$$
\begin{aligned}
& \lambda^{2}-\operatorname{Tr}(\mathbf{B K}) \lambda+\frac{1}{2}\left[\operatorname{Tr}\left(\mathbf{B} \mathbf{K}^{2}\right)-\operatorname{Tr}(\mathbf{B K})^{2}\right] \\
& \vdots \\
& \lambda^{n}-\operatorname{Tr}(\mathbf{B K}) \lambda^{n-1}+\frac{1}{2}\left[\operatorname{Tr}\left(\mathbf{B K}^{2}\right)-\operatorname{Tr}(\mathbf{B K})^{2}\right] \lambda^{n-2} \\
& \quad+\cdots+\frac{1}{2}\left[\operatorname{Tr}\left(\mathbf{B} \mathbf{K}^{2}\right)-\operatorname{Tr}(\mathbf{B K})^{2}\right] .
\end{aligned}
$$

Because $\mathbf{B K}$ is singular $\operatorname{det}(\mathbf{B K})=0$ and hence

$$
\begin{gathered}
\operatorname{det}(\mathbf{B K})=\frac{1}{2}\left[\operatorname{Tr}\left(\mathbf{B K}^{2}\right)-\operatorname{Tr}(\mathbf{B K})^{2}\right]=0, \\
\operatorname{Tr}\left(\mathbf{B} \mathbf{K}^{2}\right)=\operatorname{Tr}(\mathbf{B K})^{2}
\end{gathered}
$$


Substituting (44) into (43), then (43) becomes

$$
\begin{aligned}
& \lambda^{2}-\operatorname{Tr}(\mathbf{B K}) \lambda \longrightarrow \lambda(\lambda-\operatorname{Tr}(\mathbf{B K})) \\
& \vdots \\
& (-1)^{n} \lambda^{n}-\operatorname{Tr}(\mathbf{B K}) \lambda^{n-1} \longrightarrow(-1)^{n} \lambda^{n-1}(\lambda-\operatorname{Tr}(\mathbf{B K})) .
\end{aligned}
$$

Finally the eigenvalues of $\mathbf{B K}$ are

$$
\lambda_{1}, \ldots, \lambda_{n-1}=0 \quad \lambda_{n}=\operatorname{Tr}(\mathbf{B K})<0 .
$$

Equation (46) shows that the minimum eigenvalue of $\mathbf{B K}$ equals $\operatorname{Tr}(\mathbf{B K})$. If the eigenvalues of $\left(\mathbf{I}_{n \times n}+\tau \mathbf{B K}\right)$ are $s_{1}, \ldots, s_{n}$, then the eigenvalues of $\left(\mathbf{I}_{n \times n}+\tau \mathbf{B K}\right)^{-1}$ are $1 / s_{1}, \ldots, 1 / s_{n}$. The eigenvalues of $\left(\mathbf{I}_{n \times n}+\tau \mathbf{B K}\right)$ are given by

$$
\begin{aligned}
\tau \cdot \mathbf{B K}+\mathbf{I}_{n \times n}-s \mathbf{I}_{n \times n} & \\
& =\left[\begin{array}{cccc}
\tau b_{1} k_{1}+1-s & \tau b_{1} k_{2} & \cdots & \tau b_{1} k_{n} \\
\tau b_{2} k_{1} & \tau b_{2} k_{2}+1-s & \cdots & \tau b_{2} k_{n} \\
\vdots & \vdots & \ddots & \vdots \\
\tau b_{n} k_{1} & \tau b_{n} k_{2} & \cdots & \tau b_{n} k_{n}+1-s
\end{array}\right] .
\end{aligned}
$$

By solving (47) it can be found that

$$
\begin{gathered}
s_{1}, \ldots, s_{n-1}=1, \\
s_{n}=1+\tau \cdot \operatorname{Tr}(\mathbf{B K})=1+\tau \cdot \lambda_{\max }(\mathbf{B K}) \\
<1 /|\operatorname{Tr}(\mathbf{B K})| \rightarrow s_{n}>0 \rightarrow s_{1}, \ldots, s_{n}>0 . \\
\text { For single-input-single-output NCS we have }
\end{gathered}
$$

if $\tau<1 /|\operatorname{Tr}(\mathbf{B K})| \rightarrow s_{n}>0 \rightarrow s_{1}, \ldots, s_{n}>0$.

$$
\text { abs }(\mathbf{K B})=\operatorname{Tr}(\mathbf{B K}) ; \quad \text { then }
$$

if $\tau<1 /|\mathbf{K B}|$ and both (H.1) and (H.2) hold then the system is asymptotically stable.
This inequality can be used as a simple and fast tool for estimating the MADB in NCS and involves only single calculation.

\section{Stability Analysis Case Studies}

In general, two approaches are applied to controller design for NCSs. The first approach is to design a controller without considering time delay and then to design a communication protocol that minimizes the effects caused by time delays. The second approach is to design the controller while taking the time delay and data dropouts into account [11, 29]. The proposed method in this paper is used to estimate the MADB for predesigned control system. In this section, a number of examples are studied to demonstrate the proposed method and compare its results with the previously published cases in the literature. In particular, the results derived using the method proposed in this paper have been compared with the results using the LMI method given in [7] and with the fourth-order Pade approximation. The fourth-order Pade approximation [6] is used for the delay term in the $s$-domain and is defined as

$$
e^{-\tau s} \approx P_{d}(s)=\frac{N_{d}(s)}{D_{d}(s)}=\frac{\left(\sum_{k=0}^{n}(-1)^{k} c_{k} \tau^{k} s^{k}\right)}{\left(\sum_{k=0}^{n} c_{k} \tau^{k} s^{k}\right)} .
$$

The coefficients are given by

$$
c_{k}=\frac{((2 n-k) ! n !)}{(2 n ! k !(n-k) !)} \quad k=0,1, \ldots, n(n=4) .
$$

With the fourth-order Pade approximation, the truncation error in the time delay calculation is less than 0.0001 . The LMI-based method which has been used for the comparisons is based on using Lyapunov-Krasovskii functional and can be summarized as follows.

Corollary 6 (see [7]). For a given scalar $\tau$ and a matrix $\mathbf{K}$, if there exist matrices $\mathbf{P}>0, \mathbf{T}>0, \mathbf{N}_{i}$, and $\mathbf{M}_{i}(i=1,2,3)$ of appropriate dimension such that

$$
\left[\begin{array}{ccrr}
\mathbf{M}_{1}+\mathbf{M}_{1}^{T}-\mathbf{N}_{1} \mathbf{A}-\mathbf{A}^{T} \mathbf{N}_{1}^{T} & \mathbf{M}_{2}^{T}-\mathbf{M}_{1}-\mathbf{A}^{T} \mathbf{N}_{2}^{T}-\mathbf{N}_{1} \mathbf{B K} & \mathbf{M}_{3}^{T}-\mathbf{A}^{T} \mathbf{N}_{3}^{T}+\mathbf{N}_{1}+\mathbf{P} & \tau \mathbf{M}_{1} \\
* & -\mathbf{M}_{2}-\mathbf{M}_{2}^{T}-\mathbf{N}_{2} \mathbf{B K}-(\mathbf{B K})^{T} \mathbf{N}_{2}^{T} & -\mathbf{M}_{3}^{T}+\mathbf{N}_{2}-(\mathbf{B K})^{T} \mathbf{N}_{3}^{T} & \tau \mathbf{M}_{2} \\
* & * & \mathbf{N}_{3}+\mathbf{N}_{3}^{T}+\tau \mathbf{T} & \tau \mathbf{M}_{3} \\
* & * & * & -\tau \mathbf{T}
\end{array}\right]<0
$$

then the system (1)-(2) is exponentially asymptotically stable. With a given controller gain $\mathbf{K}$, solving the LMI in Corollary 6 using the LMI Matlab Toolbox the maximum time delay can be computed.

Example 7. The system in this example is the most widely used example in the literature and is described by the following equation:

$$
\dot{x}(t)=\left[\begin{array}{cc}
0 & 1 \\
0 & -0.1
\end{array}\right] x(t)+\left[\begin{array}{c}
0 \\
0.1
\end{array}\right] u(t) .
$$

In previous reports $[1,7]$, the feedback control is chosen to be

$$
u(t)=\left[\begin{array}{ll}
-3.75 & -11.5
\end{array}\right] x(t) .
$$


From Corollary $3,1 /\|\mathbf{B K}\|=0.8695$, so the MADB is estimated to be $0.8695 \mathrm{~s}$. Using Theorem 2 and Corollary 5 the MADB is $0.8695 \mathrm{~s}$. The same result can be obtained using the LMI method as reported in $[7,23,24,30]$. In [11, 17], the value reported for MADB is $4.5 \times 10^{-4} \mathrm{~s}$ and in [22] it is $0.0538 \mathrm{~s}$. In [29], the MADB is $0.785 \mathrm{~s}$. It has been reported in [10], where an improved Lyapunov-Krasovskii approach has been used, that the MADB is $1.0551 \mathrm{~s}$ and also $1.05 \mathrm{~s}$ reported in [23] with improved algorithm for solving the LMI. In [1], the MADB is $1.0081 \mathrm{~s}$. Using the proposed method with second order finite difference approximation we can obtain $1.13 \mathrm{~s}$ as the MADB. The system response with $0.8695 \mathrm{~s}$ time delay and $\mathbf{x}(0)=\left[\begin{array}{ll}0.1 & 0\end{array}\right]^{\mathrm{T}}$ is shown in Figure 4 which proves the system is stable with the estimated MADB.

Example 8 (see [31]). Consider

$$
\begin{gathered}
\dot{x}(t)=\left[\begin{array}{ccc}
0 & 1 & 0 \\
0 & 0 & 1 \\
0 & -2 & -3
\end{array}\right] x(t)+\left[\begin{array}{l}
0 \\
0 \\
1
\end{array}\right] u(t), \\
u(t)=\left[\begin{array}{lll}
-160 & -54-11
\end{array}\right] x(t) .
\end{gathered}
$$

For this third-order system both the LMI and our method give $0.0909 \mathrm{~s}$ as the MADB. Also with Corollary 5 the MADB is $0.0909 \mathrm{~s}$.

Example 9 (see [31]). The last example is the fourth-order model of the inverted pendulum shown in Figure 5 which is in many papers reduced to a second order system in order to verify the stability of NCSs. The pendulum mass is denoted by $m$ and the cart mass is $M$; the length of the pendulum rod is $L$. The open loop system is unstable. The states are defined as $x_{1}=x, x_{2}=\dot{x}, x_{3}=\theta$, and $x_{4}=\dot{\theta}$. The model is given by

$$
\begin{gathered}
\dot{\mathbf{x}}(t)=\left[\begin{array}{cccc}
0 & 1 & 0 & 0 \\
0 & 0 & \frac{-m g}{M} & 0 \\
0 & 0 & 0 & 1 \\
0 & 0 & \frac{(M+m) g}{M L} & 0
\end{array}\right] \mathbf{x}(t)+\left[\begin{array}{c}
0 \\
\frac{1}{M} \\
0 \\
\frac{-1}{M L}
\end{array}\right] u(t), \\
\mathbf{y}(t)=\left[\begin{array}{c}
x \\
\theta
\end{array}\right]=\left[\begin{array}{llll}
1 & 0 & 0 & 0 \\
0 & 0 & 1 & 0
\end{array}\right] \mathbf{x}(t) .
\end{gathered}
$$

The parameters used are $M=2 \mathrm{~kg}, m=0.1 \mathrm{~kg}$, and $L=$ $0.5 \mathrm{~m}$. Then the linear model becomes

$$
\dot{x}(t)=\left[\begin{array}{cccc}
0 & 1.000 & 0 & 0 \\
20.601 & 0 & 0 & 0 \\
0 & 0 & 0 & 1 \\
-0.4905 & 0 & 0 & 0
\end{array}\right] x(t)+\left[\begin{array}{c}
0 \\
-1 \\
0 \\
0.5
\end{array}\right] u(t) .
$$

Using the LQR Matlab function with $\mathbf{Q}=\mathbf{I}$ and $\mathbf{R}=1$, the controller is given by

$$
\mathbf{K}_{\mathrm{LQR}}=\left[\begin{array}{llll}
52.1238 & 11.5850 & 1.000 & 2.7252
\end{array}\right] .
$$

Using the LMI method the MADB is $0.0978 \mathrm{~s}$ and our method gives $0.0978 \mathrm{~s}$ using Theorem 2 and Corollary 5 . We noted that there is a good agreement between our method

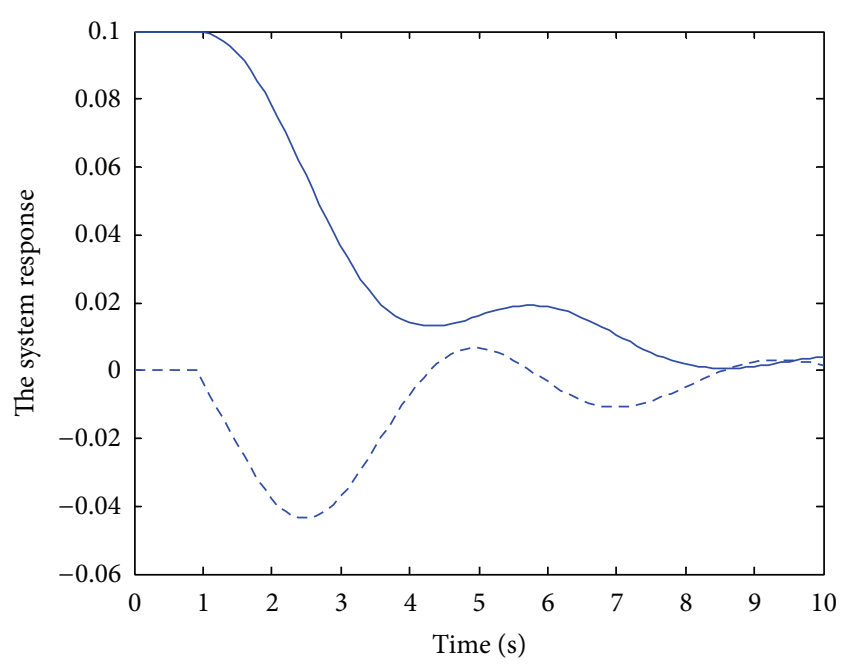

FIgURE 4: The response of the system in Example 7 with $0.8695 \mathrm{~s}$ delay.

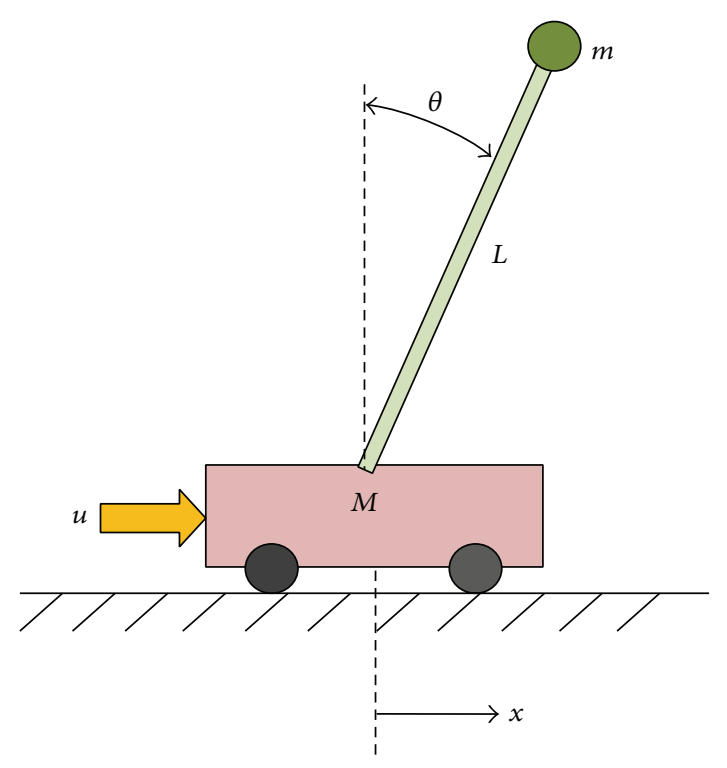

FIgURE 5: The inverted pendulum on a cart.

and the LMI method because $\tau$ is small enough to make the finite difference approximation hold. The system response with $0.0978 \mathrm{~s}$ time delay and with $x=0$ and $\theta=0.1$ is shown in Figure 6 which shows the system is stable. Many examples have been studied to compare the results obtained using the method proposed in this paper with the results obtained using the LMI method [7] and the fourthorder Pade approximation method. The calculation results are summarized in Table 1 along with the simulation based MADB.

Remarks. From Table 1, it can be seen that the proposed new method can give values of MADB similar to the values obtained using the LMI method and the other methods; however, the method proposed in this paper has a much 
TABLE 1: The MADB (seconds) using the proposed method with 1st, 2nd, and 3rd order finite difference approximation for the delay term, the LMI method, the fourth-order Pade approximation method, and the simulation based method.

\begin{tabular}{|c|c|c|c|c|c|c|}
\hline & & te difference & & The IMI & Pade annroximation & Simulation based \\
\hline & 1st order & 2nd order & 3rd order & 1110 LIVIT & 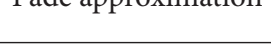 & - \\
\hline 1 & 0.8695 & 0.8427 & 1.1321 & 0.8696 & 1.1672 & 1.180 \\
\hline 2 & 0.1000 & 0.0995 & 0.1421 & 0.1000 & 0.1475 & 0.149 \\
\hline 3 & 0.0100 & 0.0099 & 0.0149 & 0.0100 & 0.0156 & 0.0157 \\
\hline 4 & 0.1428 & 0.1385 & 0.1808 & 0.1429 & 0.1855 & 0.1860 \\
\hline 5 & 0.8217 & 0.8489 & 0.9085 & 0.8217 & 0.9091 & 0.9140 \\
\hline 6 & 0.5000 & 0.4816 & 0.6303 & 0.5000 & 0.6474 & 0.6510 \\
\hline 7 & 0.9940 & 0.9940 & 0.9960 & 0.9940 & 0.9960 & 0.9970 \\
\hline 8 & 0.0856 & 0.0854 & 0.1192 & 0.0856 & 0.1230 & 0.1230 \\
\hline 9 & 0.0906 & 0.0919 & 0.1251 & 0.0909 & 0.1284 & 0.1285 \\
\hline 10 & 0.0416 & 0.0400 & 0.0496 & 0.0416 & 0.0505 & 0.0505 \\
\hline
\end{tabular}

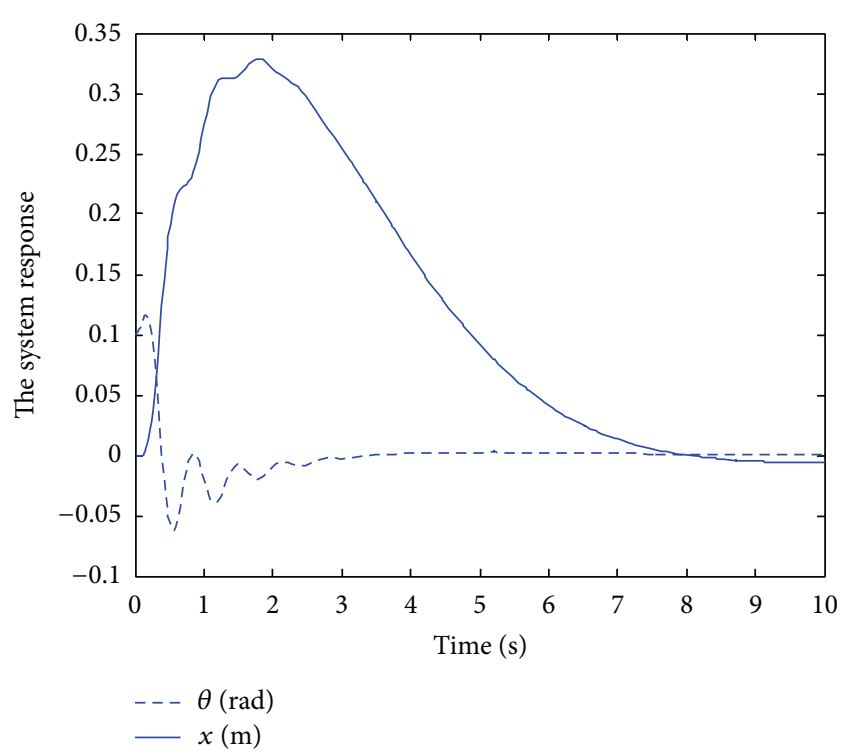

Figure 6: The response of the system in Example 9 with $0.0978 \mathrm{~s}$ delay.

simpler procedure, and it should have no difficulties for practical design engineers to accept this approach. Clearly, the $\mathrm{MADB}$ with the first-order finite difference approximation is comparable with the LMI method. Furthermore, we found good agreement between the third-order finite difference approximation and the fourth-order Pade approximation. The simulation based results for the MADB show that the estimated MADB through the proposed method sufficiently achieves the system stability. A simple controller design method has been developed by the authors based on the method presented in this paper. In the controller design method a stabilizing controller can be derived for a given network time delay. In all the case studies or examples, only linear system examples are given. The method is limited to linear systems only. The authors are now working on extending the methods to nonlinear systems, such as, multiconverter and inverter system and engine and electrical power generation systems $[32,33]$.
The application of the finite difference approximation for representing the time delay is not new but we found in this paper that using higher order approximations can sufficiently represent the time delay linear system. From Table 1 it can be concluded that using the first order approximation the estimated MADB is comparable with the other two methods. This is because the derivation of the linear model from the nonlinear model is based on neglecting the higher order derivative terms. In some cases we need to use the higher derivative terms for the time delay in order to achieve more accurate results for the MADB. The current research is to derive sufficient conditions for applying the method in order to find the tolerance of the estimated MADB.

\section{Concluding Remarks}

The main contribution of the paper is to have derived a new method for estimating the maximum time delay in NCSs. The most attractive feature of the new method is that it is a simple approach and easy to be applied, which can be easily interpreted to design engineers in industrial sectors. The results obtained in this method are compared with those obtained through the methods introduced in the literature. The method has demonstrated its merits in using less computation time due to its simple structure and giving less conservative results while showing good agreement with other methods. The method is limited to linear systems only and the work for extending the method for a class of nonlinear systems is on-going.

\section{Conflict of Interests}

The authors declare that there is no conflict of interests regarding the publication of this paper.

\section{References}

[1] X. Jiang, Q. L. Han, S. Liu, and A. Xue, "A new $H_{\infty}$ stabilization criterion for networked control systems," IEEE Transactions on Automatic Control, vol. 53, no. 4, pp. 1025-1032, 2008. 
[2] G. C. Walsh, H. Ye, and L. Bushnell, "Stability analysis of networked control systems," in Proceedings of the American Control Conference (ACC '99), vol. 4, pp. 2876-2880, San Diego, Calif, USA, June 1999.

[3] G. C. Walsh, H. Ye, and L. G. Bushnell, "Stability analysis of networked control systems," IEEE Transactions on Control Systems Technology, vol. 10, no. 3, pp. 438-446, 2002.

[4] M. S. Mahmoud, Robust Control and Filtering For Time Delay systems, Marcel Dekker, New York, NY, USA, 2000.

[5] M. S. Mahmoud and A. Ismail, "Role of delays in networked control systems," in Proceedings of the 10th IEEE International Conference on Electronics, Circuits and Systems (ICECS '03), vol. 1, pp. 40-43, December 2003.

[6] J. E. Marshall, H. Gorecki, A. Korytowski, and K. Walton, Time-Delay Systems: Stability and Performance Criteria with Applications, Ellis Horwood, 1992.

[7] D. Yue, Q.-L. Han, and C. Peng, "State feedback controller design of networked control systems," IEEE Transactions on Circuits and Systems II: Express Briefs, vol. 51, no. 11, pp. 640644, 2004.

[8] P.-L. Liu, "Exponential stability for linear time-delay systems with delay dependence," Journal of the Franklin Institute, vol. 340, no. 6-7, pp. 481-488, 2003.

[9] B. Tang, G. P. Liu, and W. H. Gui, "Improvement of state feedback controller design for networked control systems," IEEE Transactions on Circuits and Systems, vol. 55, no. 5, pp. 464-468, 2008.

[10] J. Sun, G. Liu, and J. Chen, "State feedback stabilization of networked control systems," in Proceedings of the 27th Chinese Control Conference (CCC '08), pp. 457-461, Kunming, China, July 2008.

[11] W. Zhang, M. S. Branicky, and S. M. Phillips, "Stability of networked control systems," IEEE Control Systems Magazine, vol. 21, no. 1, pp. 84-97, 2001.

[12] X. Li and C. E. de Souza, "Delay-dependent stability of linear time-delay systems: an LMI approach," IEEE Transactions on Automatic Control, vol. 42, no. 8, pp. 1144-1148, 1997.

[13] W. Min and H. Yong, "Improved stabilization method for networked control systems," in Proceedings of the 26th Chinese Control Conference (CCC '07), pp. 544-548, Hunan, China, July 2007.

[14] X.-L. Zhu and G.-H. Yang, "New results on stability analysis of networked control systems," in Proceedings of the American Control Conference, pp. 3792-3797, Seattle, Wash, USA, June 2008.

[15] M. Jun and M. G. Safonov, "Stability analysis of a system with time-delay states," in Proceeding of the American Control Conference, Chicago, Ill, USA, June 2000.

[16] K. Kim, "A delay-dependent stability criterion in time delay system," Journal of the Korean Society for Industrial and Applied Mathematics, vol. 9, no. 2, pp. 1-11, 2005.

[17] M. S. Branicky, S. M. Phillips, and W. Zhang, "Stability of networked control systems: explicit analysis of delay," in Proceedings of the American Control Conference, pp. 2352-2357, Chicago, Ill, USA, June 2000.

[18] L. Xie, J.-M. Zhang, and S.-Q. Wang, "Stability analysis of networked control system," in Proceedings of 1st International Conference on Machine Learning and Cybernetics, pp. 757-759, Beijing, China, November 2002.

[19] A. Luis and P. J. Montestruque, "Model-based networked control systems- necessary and sufficient conditions for stability," in Proceedings of the 10th Mediterranean Conference on Control and Automation, pp. 1-58, Lisbon, Portugal, July 2002.

[20] Y. Sun and N. H. El-Farra, "Quasi-decentralized model-based networked control of process systems," Computers \& Chemical Engineering, vol. 32, no. 9, pp. 2016-2029, 2008.

[21] J. Nilsson, Real-time control systems with delays [Ph.D. thesis], Institute of Technology, Lund, Sweden, 1998.

[22] H. S. Park, Y. H. Kim, D.-S. Kim, and W. H. Kwon, "A scheduling method for network-based control systems," IEEE Transactions on Control Systems Technology, vol. 10, no. 3, pp. 318-330, 2002.

[23] Y. Zhang, Q. Zhong, and L. Wei, "Stability analysis of networked control systems with communication constraints," in Proceedings of the Chinese Control and Decision Conference, pp. 335-339, July 2008.

[24] Y. Zhang, Q. Zhong, and L. Wei, "Stability analysis of networked control systems with transmission delays," in Proceedings of the Chinese Control and Decision Conference, pp. 340-343, July 2008.

[25] J. Wang, Ü. Kotta, and J. Ke, “Tracking control of nonlinear pneumatic actuator systems using static state feedback linearisation of input/output map," Proceedings of the Estonian Academy of Sciences: Physics, Mathematics, vol. 56, no. 1, pp. 4766, 2007.

[26] D. P. Goodall and J. Wang, "Stabilization of a class of uncertain nonlinear affine systems subject to control constraints," International Journal of Robust and Nonlinear Control, vol. 11, no. 9, pp. 797-818, 2001.

[27] J. Wang, J. Pu, P. R. Moore, and Z. Zhang, "Modelling study and servo-control of air motor systems," International Journal of Control, vol. 71, no. 3, pp. 459-476, 1998.

[28] C. D. Meyer, Matrix Analysis and Applied Linear Algebra, SIAM, 2000.

[29] F. Yang and H. Fang, "Control strategy design of networked control systems based on maximum allowable delay bounds," in Proceedings of the IEEE International Conference on Control and Automation (ICCA '07), pp. 794-797, Guangzhou, China, June 2007.

[30] P. Naghshtabrizi, Delay impulsive systems: a framework for modeling networked control systems [Ph.D. thesis], University of California, Los Angeles, Calif, USA, 2007.

[31] K. Ogata, Modern Control Engineering, Prentice Hall, New York, NY, USA, 3rd edition, 1997.

[32] J. L. Wei, J. Wang, and Q. H. Wu, "Development of a multisegment coal mill model using an evolutionary computation technique," IEEE Transactions on Energy Conversion, vol. 22, no. 3, pp. 718-727, 2007.

[33] N. Jia, J. Wang, K. Nuttall et al., "HCCI engine modeling for realtime implementation and control development," IEEE/ASME Transactions on Mechatronics, vol. 12, no. 6, pp. 581-589, 2007. 


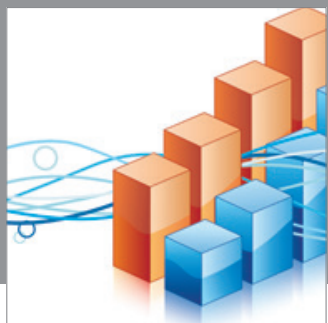

Advances in

Operations Research

mansans

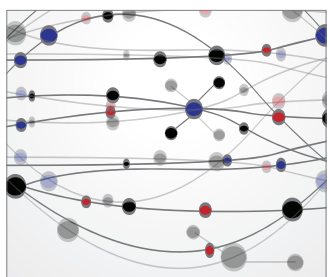

The Scientific World Journal
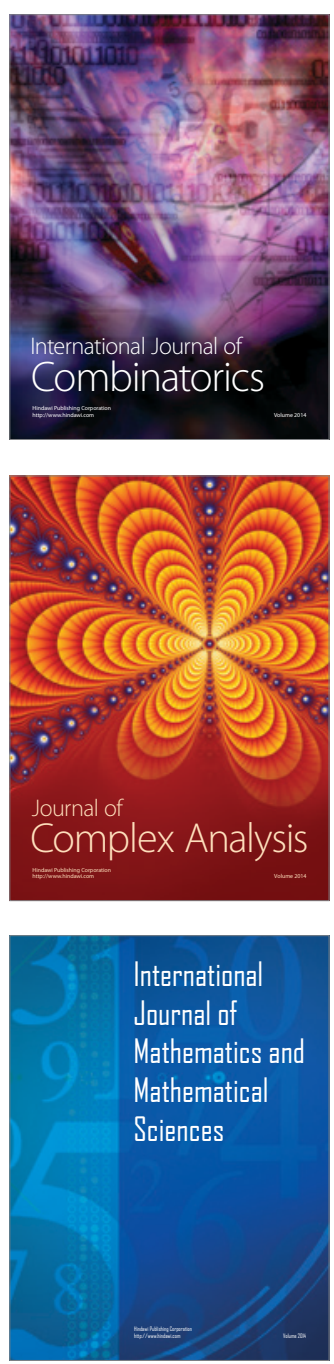
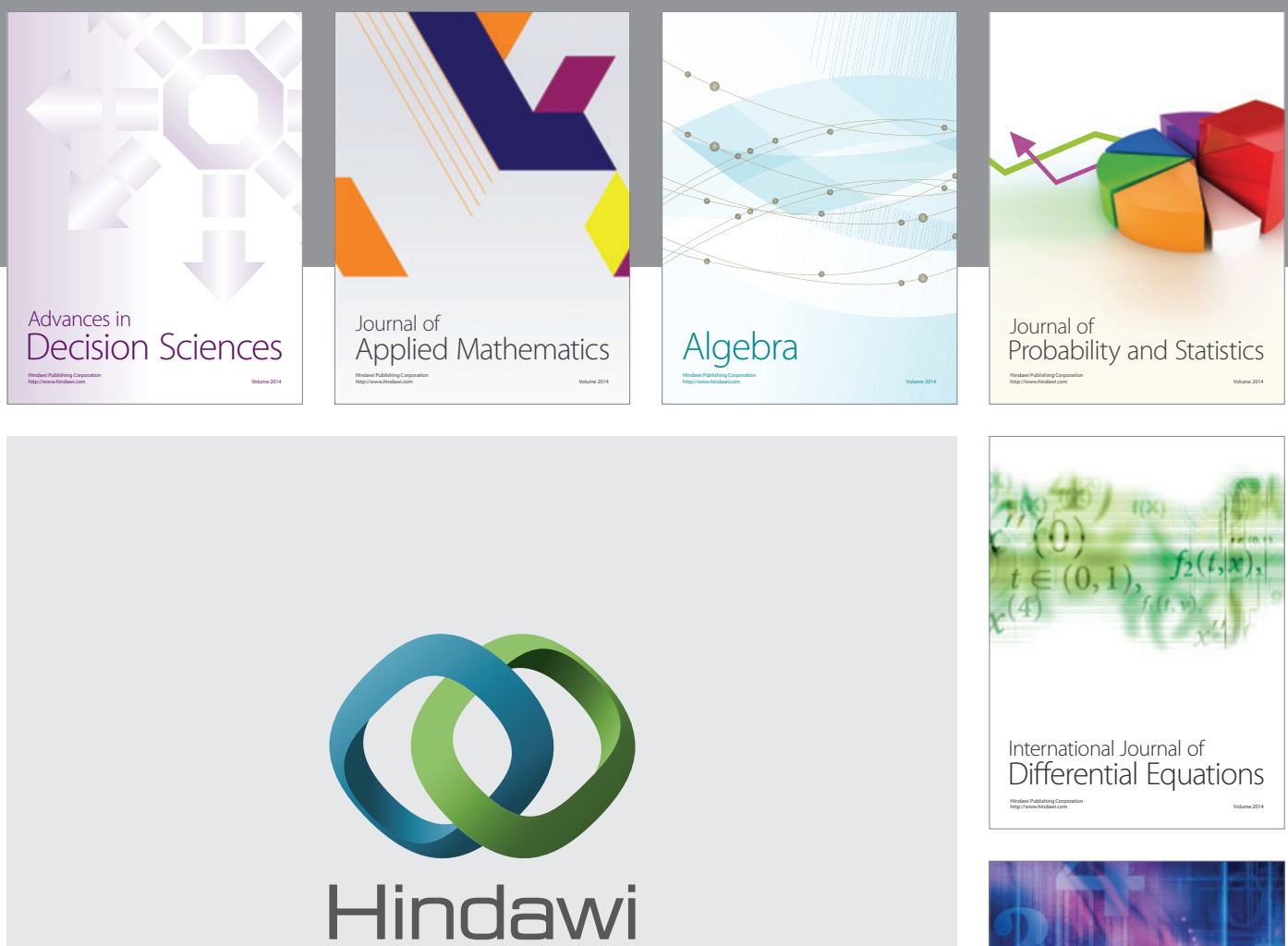

Submit your manuscripts at http://www.hindawi.com
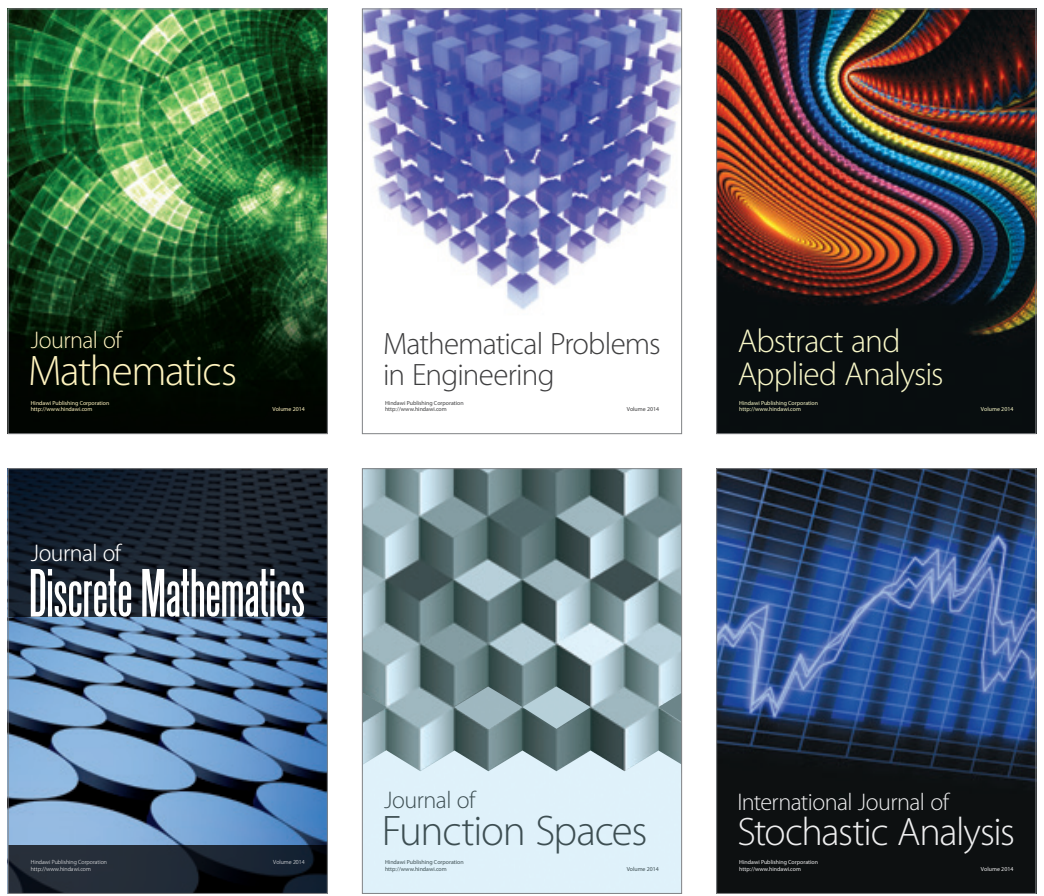

Journal of

Function Spaces

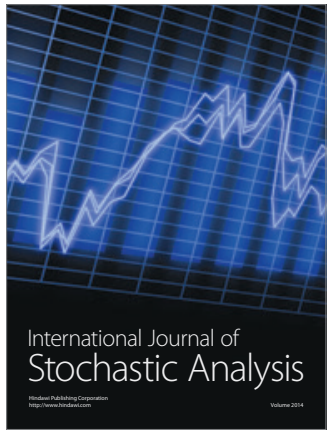

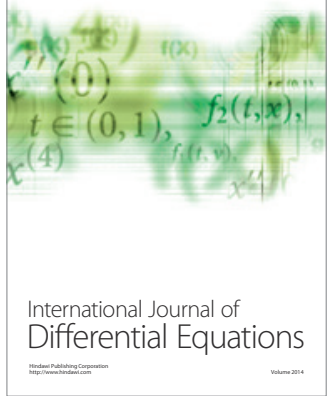
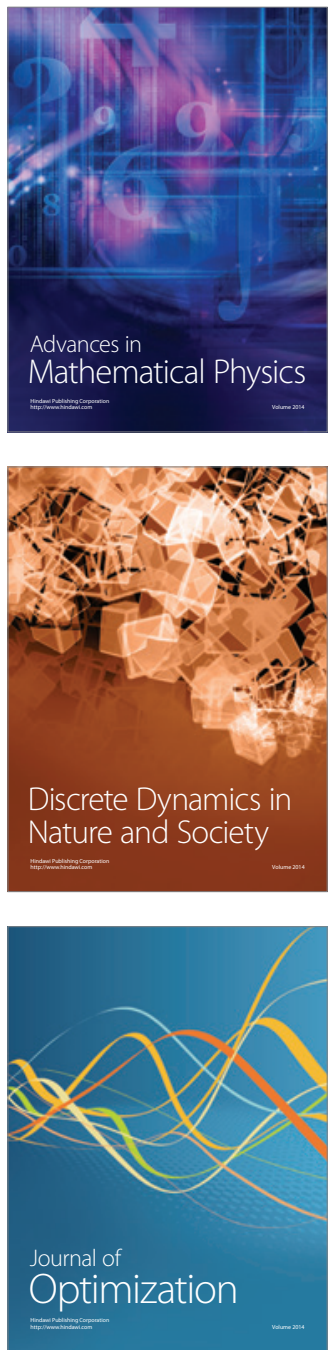